\title{
Infrared reflection spectra of forsterite crystal
}

\author{
H. Sogawa ${ }^{1}$, C. Koike ${ }^{1}$, H. Chihara ${ }^{1,2}$, H. Suto ${ }^{3}$, S. Tachibana ${ }^{4}$, A. Tsuchiyama ${ }^{2}$, and T. Kozasa ${ }^{5}$ \\ 1 Kyoto Pharmaceutical University, Yamashina, Kyoto 607-8412, Japan \\ e-mail: koike@mb . kyoto-phu.ac.jp \\ 2 Department of Earth and Space Science, Osaka University, Toyonaka, Osaka, Japan \\ 3 Subaru Telescope, 650 North Aohoku Place, Hilo, Hawaii 96720, USA \\ 4 Department of Earth and Planetary Science, The University of Tokyo, Japan \\ 5 Division of Earth and Planetary Sciences, Graduate School of Science, Hokkaido University, Sapporo 060-0810, Japan
}

Received 26 June 2004 / Accepted 3 January 2006

\begin{abstract}
Polarized infrared reflectance of large single crystals of forsterite was measured for each crystal axis at frequencies between $5000 \mathrm{~cm}^{-1}$ and $100 \mathrm{~cm}^{-1}$ with a resolution of $1 \mathrm{~cm}^{-1}$. The reflectance spectra were analyzed based on classical dispersion theory. A set of oscillator parameters for crystalline axes of forsterite was obtained. Those parameters are used to derive optical constants of forsterite, which are important for analyses of observed spectra of astronomical objects and laboratory spectra of particle samples. Calculations of absorption for small particles using the bulk optical constants are compared with mid-infrared and far-infrared absorption spectra measured in the laboratory.
\end{abstract}

Key words. methods: laboratory - ISM: dust, extinction - stars: circumstellar matter - ISM: lines and bands

\section{Introduction}

Observations obtained using Infrared Space Observatory (ISO) and ground-based telescopes have revealed the existence of crystalline silicates in the circumstellar region of evolved and young stars as well as in comets and meteorites in the solar system (e.g. Waters et al. 1996; Waelkens et al. 1996; Cesarsky et al. 2000; Molster et al. 2002; Honda et al. 2003). Midinfrared and far-infrared spectra of those objects show emission features that have been identified as crystalline silicates such as magnesium-rich olivines $\left(\mathrm{Mg}_{2 x} \mathrm{Fe}_{2(1-x)} \mathrm{SiO}_{4}\right)$ or pyroxenes $\left(\mathrm{Mg}_{x} \mathrm{Fe}_{1-x} \mathrm{SiO}_{3}\right)$.

That identification is made through comparison between infrared emission features observed for astronomical objects and laboratory absorption spectra of powdered samples. However, in the laboratory, the powdered samples are usually embedded in a matrix of $\mathrm{KBr}$ or polyethylene and the measured spectrum is not identical to that of the grains in vacuum. Moreover, different conditions of preparing sample pellets readily yield different spectra because the absorption spectra can be affected by the grains' size and shape distributions as well as by the degree of dispersion in the matrix (e.g., Koike \& Shibai 1994; Hofmeister et al. 2000, and references therein).

On the other hand, optical constants of bulk crystal samples provide more definite and reliable standards for the study of crystalline dust. Absorption spectra of particles are calculable by assuming the size and shape distributions of particles.
Forsterite $\left(\mathrm{Mg}_{2} \mathrm{SiO}_{4}\right)$ is a magnesium-rich end-member of the olivine group. Numerous studies have investigated lattice vibrations of forsterite (Servoin \& Piriou 1973; Iishi 1978; Hofmeister 1987; Reynard 1991; Fabian et al. 2001). The oscillator parameters for the lattice vibrations, from which the optical constants can be derived, have also been obtained (Servoin \& Piriou 1973; Iishi 1978; Fabian et al. 2001). However, no experiment so far has used a sufficiently high resolution and a high $\mathrm{S} / \mathrm{N}$ ratio covering the mid-infrared and far-infrared wavelengths for a single-crystalline sample, especially at wavelengths that are longer than $40 \mu \mathrm{m}$.

We measured reflectance spectra of single-crystals of synthesized forsterite and determined the oscillator parameters based on the classical oscillator model to derive new optical constants of forsterite at mid-infrared and far-infrared wavelengths. Absorption spectra of small particles calculated using optical constants derived from these parameters were subsequently compared with laboratory spectra.

\section{Experiment}

A forsterite single crystal was synthesized using the Czochralski method (Takei \& Kobayashi 1974). Three pieces of centimeter-sized forsterite crystals were cut from the single crystal and were used for the experiment. Crystalline axes of each bulk sample were determined using X-ray precession method. Surface planes for the reflectance measurement were selected to maximize the surface area for crystalline samples. 
They were cut perpendicular to the crystalline axes and polished. Reflections polarized parallel to the $\mathrm{a}, \mathrm{b}$, and c axes were measured respectively on the (010), (001), and (100) faces.

Experiments were conducted at room temperature. Infrared spectra from $5000 \mathrm{~cm}^{-1}$ to $100 \mathrm{~cm}^{-1}$ with a resolution of $1 \mathrm{~cm}^{-1}$ were obtained using an FTIR spectrometer (Nicolet Nexus 670) with an internal Ever Glo IR source and a DTGS (/KBr or $/ \mathrm{PE}$ windows) detector. The beam size of the measurement was $\phi=5 \mathrm{~mm}$. A $\mathrm{KBr}$ beamsplitter was used to measure reflectance at frequencies between 5000 and $400 \mathrm{~cm}^{-1}$; and a solid substrate beamsplitter was used at frequencies between 400 and $100 \mathrm{~cm}^{-1}$. The incident light entered at $10^{\circ}$ from the normal plane and the electric field of the light was polarized along the crystal axis and perpendicular to the plane of incidence that was defined by the incident and reflected light beams. A wire-grid polarizer with a KRS5 substrate was used at frequencies from $5000 \mathrm{~cm}^{-1}$ to $230 \mathrm{~cm}^{-1}$; a polarizer with polyethylene substrate was used at frequencies from $230 \mathrm{~cm}^{-1}$ to $100 \mathrm{~cm}^{-1}$.

The reflection spectra of several measurements were averaged for each axis. The systematic difference between the averaged spectrum and one measurement was well within $\sim 1 \%$. The reflectance of the gold mirror used for calibration was assumed as $100 \%$.

\section{Results}

The obtained reflection spectra for the three different crystallographic axes are shown in Fig. 1. They closely resemble the spectra shown in the investigation of Reynard (1991), which includes the latest reflectance measurements of single-crystalline forsterite with a resolution of $1 \mathrm{~cm}^{-1}$ in $450-5000 \mathrm{~cm}^{-1}$ and a resolution of $4 \mathrm{~cm}^{-1}$ in $100-700 \mathrm{~cm}^{-1}$. However, our highresolution spectra with a higher $\mathrm{S} / \mathrm{N}$ ratio at low frequency show the $\sim 150 \mathrm{~cm}^{-1}$ feature in $\mathrm{B}_{2 \mathrm{u}}$ and the $\sim 200 \mathrm{~cm}^{-1}$ feature in $\mathrm{B}_{3 \mathrm{u}}$ more clearly.

There are 35 IR active $\left(9 \mathrm{~B}_{1 \mathrm{u}}, 13 \mathrm{~B}_{2 \mathrm{u}}\right.$, and $\left.13 \mathrm{~B}_{3 \mathrm{u}}\right)$ optical modes described from group analysis (Iishi 1978). The reflectance spectra show 31 features $\left(8 \mathrm{~B}_{1 \mathrm{u}}, 12 \mathrm{~B}_{2 \mathrm{u}}\right.$, and $\left.11 \mathrm{~B}_{3 \mathrm{u}}\right)$ with good $\mathrm{S} / \mathrm{N}$ ratios. An additional small peak of reflectance at $\sim 474 \mathrm{~cm}^{-1}$ in $B_{3 u}$ is apparent. However, it was not taken into account for the fitting procedure to determine the oscillator parameters because it is too small to fit the model properly.

Identification of the IR vibration modes in previous works are summarized in Reynard (1991). Most vibration modes identified by Reynard are observed in our measurements; nondetected bands are the mode related to the feature at $\omega=$ $420 \mathrm{~cm}^{-1}$ in $B_{3 u}$ and the mode at $\omega=370 \mathrm{~cm}^{-1}$ in $B_{1 u}$. The reflectance peak at $400-460 \mathrm{~cm}^{-1}$ in $\mathrm{B}_{3 \mathrm{u}}$ is interpreted as the combination of two modes in Reynard (1991), Hofmeister (1987), and Servoin \& Piriou (1973), whereas it is identified as a single mode in Iishi (1978). In analyses of the reflectance spectra of $\mathrm{Mg}$-rich olivine, the corresponding peak is regarded as arising from a single mode; all vibration modes (13 modes) predicted by the group analysis are identified (Fabian et al. 2001). Therefore, we interpret the peak as a single mode. The mode at $\sim 370 \mathrm{~cm}^{-1}$ in $\mathrm{B}_{1 \mathrm{u}}$ is clearly visible in the reflectance spectra of Mg-rich olivines (Reynard 1991; Fabian et al. 2001).

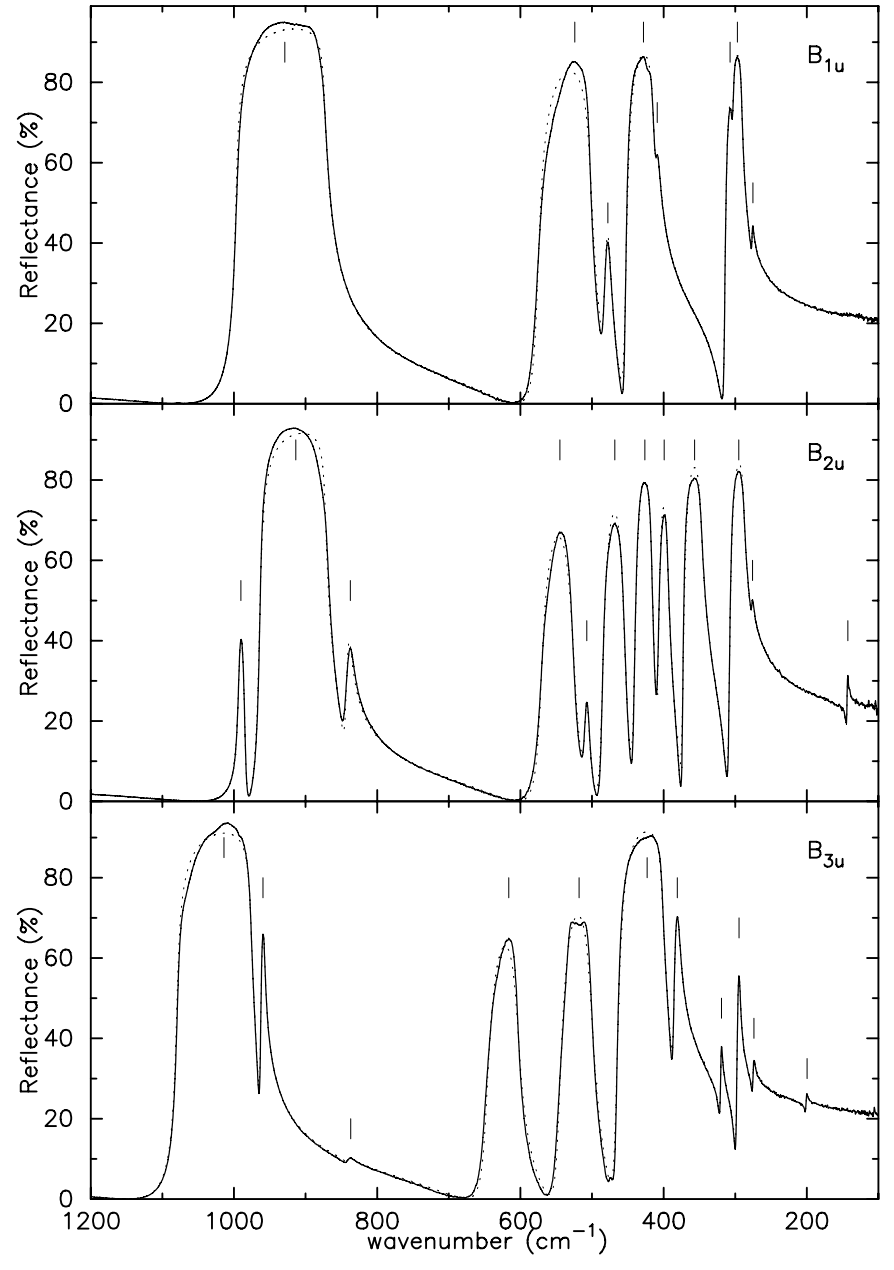

Fig. 1. Solid line: Reflectivity for three different crystallographic axes of forsterite. Data of $\mathrm{B}_{1 \mathrm{u}}, \mathrm{B}_{2 \mathrm{u}}$, and $\mathrm{B}_{3 \mathrm{u}}$ were taken respectively from (100), (001), and (010) faces. Dotted line: Best fit calculated using the multiple oscillators model. Vertical dashes indicate the IR vibration bands.

However, our reflectance spectra of forsterite as well as the spectra in Reynard (1991) and Servoin \& Piriou (1973) show no evident features. We assume that the contribution of the mode is negligible for forsterite.

The feature at $\omega=307 \mathrm{~cm}^{-1}$ observed in our $B_{1 \mathrm{u}}$ spectrum is explained using the longitudinal optic frequency at $305 \mathrm{~cm}^{-1}$ in $B_{2 u}$ in Reynard (1991). However, because we can observe the same profile in both reflectance measurements of the (100) face and the (010) face (not shown here), we identify the feature at $\omega=307 \mathrm{~cm}^{-1}$ as a band in $\mathrm{B}_{1 \mathrm{u}}$.

The existence of the mode at $\sim 200 \mathrm{~cm}^{-1}$ in $\mathrm{B}_{1 \mathrm{u}}$ is suggested by Oehler \& Günthard (1969) and Fabian et al. (2001) based on measurements of the powdered or polycrystalline samples. The corresponding feature is not observed in our measurements for the forsterite single-crystal. The contribution from the mode at $\sim 200 \mathrm{~cm}^{-1}$ in $\mathrm{B}_{3 \mathrm{u}}$ might be misidentified with the mode in $\mathrm{B}_{1 \mathrm{u}}$ in the case of powdered and polycrystalline samples. 
The spectra were analyzed using classical dispersion theory. We used the dispersion relation with the multiple oscillator model, which is given as

$\epsilon(\omega)=\epsilon_{0}+\sum_{i} \frac{\omega_{p i}^{2}}{\omega_{i}^{2}-\omega^{2}-i \gamma_{i} \omega}$,

where $\epsilon(\omega)$ is a complex dielectric function for the frequency $\omega, \epsilon_{0}$ is the dielectric constant at the high-frequency limit, and $\omega_{i}, \omega_{p i}^{2}$, and $\gamma_{i}$ respectively represent the resonance frequency, the oscillator strength, and the damping constant of the $i$ th oscillator (Bohren \& Huffman 1983).

The reflectance $R$ at the incident angle of $\theta$ is calculated from $\epsilon$ as

$R(\omega)=\left|\frac{\sqrt{\epsilon(\omega)-\sin ^{2} \theta}-\cos \theta}{\sqrt{\epsilon(\omega)-\sin ^{2} \theta}+\cos \theta}\right|^{2}$

for the incident electric field perpendicular to the incident plane.

Values of oscillator parameters derived from the leastsquares fitting to the reflection spectra are tabulated in Table 1.

The dotted lines in Fig. 1 show the reflectance calculated with the parameters. The fit is fairly good, and especially good for frequencies lower than $\sim 300 \mathrm{~cm}^{-1}$, though there are small discrepancies for the intensities and peak positions of prominent features. These spectra are very delicate for subtle amounts of inaccuracy in preparing surfaces polished perpendicular to the crystalline axis ${ }^{1}$.

The difference between the refractive index in the visible (1.651 in $\mathrm{B}_{1 \mathrm{u}}, 1.635$ in $\mathrm{B}_{2 \mathrm{u}}, 1.670$ in $\mathrm{B}_{3 \mathrm{u}}$; Deer et al. 1996) and the value of the square root of the fitted $\epsilon_{0}$ is within $\sim 0.5 \%$ in each crystal axis.

\section{Comparison with absorption spectra of fine particles}

We can calculate absorption spectrum of small particles using the bulk optical constants estimated from the oscillator parameters. In this study, that calculation will not be compared with astronomical spectra because the particle shapes, along with the composition and temperature of dust grains, affect the appearance of emission features. Instead, the spectrum is compared with absorption spectra of small particles ground from bulk crystalline samples.

The thick line in Fig. 2 shows the mass absorption coefficients (MACs) of forsterite obtained from transmittance measurements of forsterite powder samples (Koike et al. 2003).

\footnotetext{
${ }^{1}$ For reference, we measured the reflections whose electric field was polarized parallel to the $a$-axis on the (001) face, the $b$-axis on (100), and the $c$-axis on (010) in the mid-infrared wavelengths. The reflection spectra of $a$-axis measured on (001) show a small ( $2 \%$ in reflectivity) dip at $\sim 994 \mathrm{~cm}^{-1}$ which is attributed to the longitudinal optic (LO) component of $\mathrm{B}_{1 \mathrm{u}}$. This dip suggests that the polished face is not perfectly perpendicular to the $c$-axis. Similarly, reflection spectra that are polarized parallel to the $c$-axis measured on the (010) face also show a small dip at $\sim 964 \mathrm{~cm}^{-1}$, which is attributed to the LO component of $\mathrm{B}_{2 \mathrm{u}}$.
}

Table 1. Oscillator Parameters for IR bands of forsterite crystal.

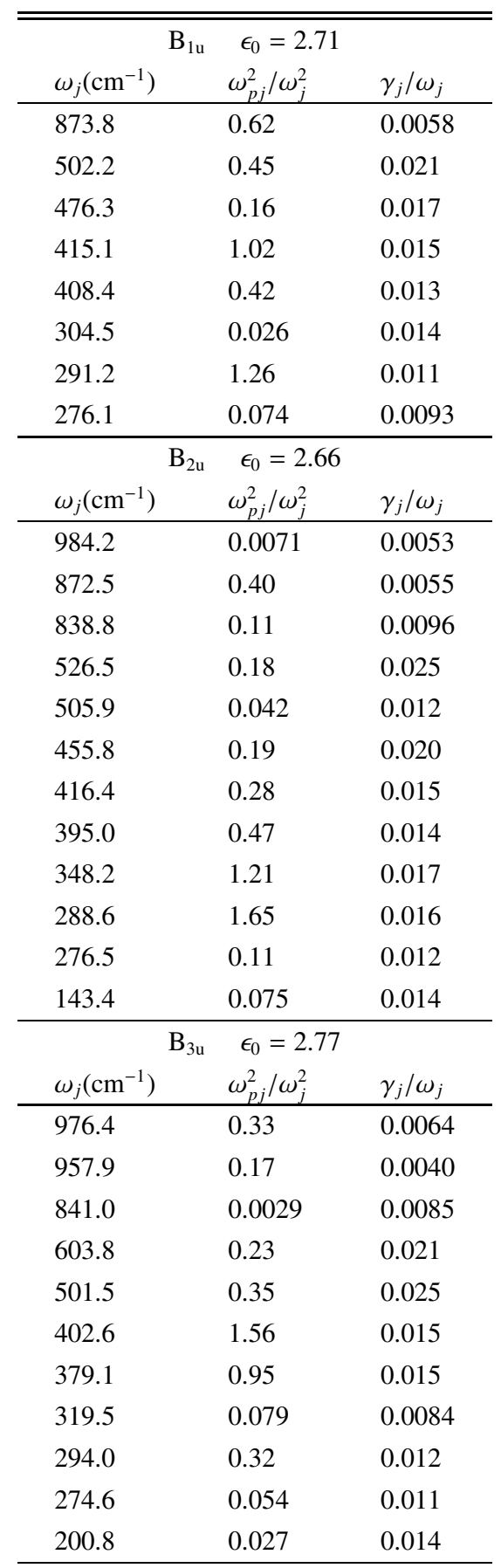

Powder samples were produced by grinding the bulk crystal that was synthesized using the same method as that used for producing bulk samples for reflectance measurement. The powder is embedded in a $\mathrm{KBr}$ matrix for spectroscopy in the wavelength shorter than $25 \mu \mathrm{m}$ and in a polyethylene sheet for spectroscopy in the wavelength longer than $25 \mu \mathrm{m}$.

Recent studies have shown that the shape of the particles of crystal olivines (including forsterite and fayalite) has a salient effect on the positions and intensities of prominent resonance features and that spectra calculated for spherical particles differ greatly from laboratory spectra. The spectrum calculated for a 


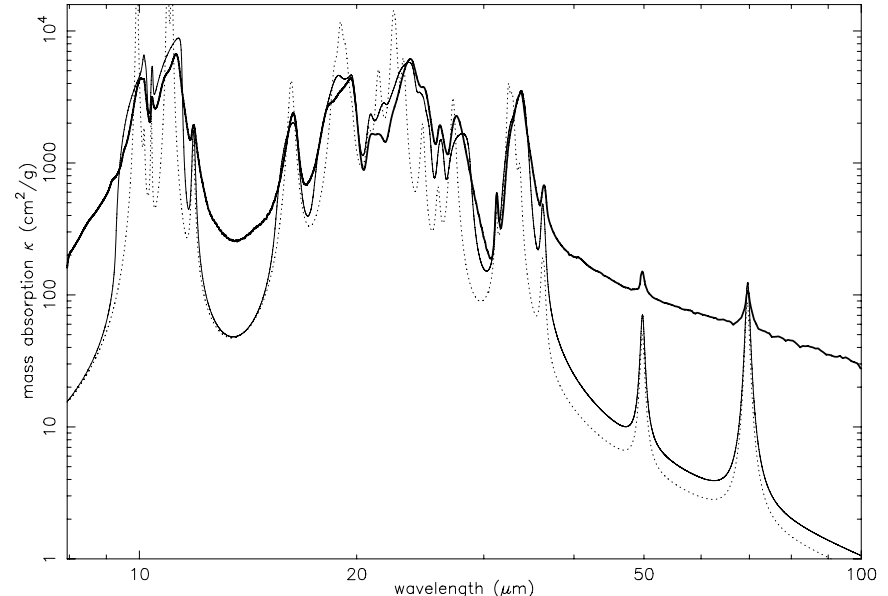

Fig. 2. Comparison of absorption spectra calculated from optical constants of bulk forsterite for a spherical shape (dotted line) and a $\mathrm{CDE}$ (thin line) with the MAC spectrum measured in the laboratory for forsterite particles (thick line; Koike et al. 2003).

continuous distribution of ellipsoids (CDE) model, which consists of various shapes of triaxial ellipsoids, is a fairly good approximation for laboratory spectra (Jäger et al. 1998; Fabian et al. 2001; Suto et al. 2002; Min et al. 2003).

Spectra calculated for the CDE are represented with the thin line in Fig. 2; in the calculation, the formula for refractive indices of $\mathrm{KBr}$ in Heaton (1970) and $n=1.46$ for a refractive index of polyethylene is assumed. For mass density of forsterite, $3.23 \mathrm{~g} / \mathrm{cm}^{3}$ is used. For comparison, the absorption spectra calculated for spherical particles are also shown by a dotted line. The CDE model reasonably reproduces the measured spectrum, but the continuum level of experimental spectra is higher than those of the CDE model, probably because of the effect of the light scattering from the aggregation of forsterite particles in sample pellets for transmittance measurement.

Figure 3 shows the difference of MACs among powder samples. Different conditions of sample preparation (e.g., the material of mortars, time and power spent for grinding) produce the different shape distributions of fine particles and consequently create different spectra. The MACs for spherical shape and the CDE are overlapped in the figure. As also seen in Fig. 2, the feature about $19 \mu \mathrm{m}$ for the CDE is not observed in either of the powder samples. The spectral profile at $20-22 \mu \mathrm{m}$ and the peak wavelength of the feature $\sim 28 \mu \mathrm{m}$ is also different from experimental results. Several reasons are given for these discrepancies.

With respect to the transmittance measurements of the powder samples, if the particle size is too large to transmit light at a certain wavelength, the profile of the spectral feature changes and might produce artifacts (Hofmeister et al. 2000). Scanning Electron Microscope (SEM) images of particles in our sample pellets show that the most particles are $d=0.2 \sim 0.4 \mu \mathrm{m}$, but a few large particles of $d \sim 1 \mu$ m exist. Additionally, as expected from the continuum level of mass absorption spectra, micrometer-sized aggregates of particles exist in the samples. Figure 4 shows the calculation of $2 \pi k / \lambda$ for three crystalline axes, where $k$ is the imaginary part of the complex index of

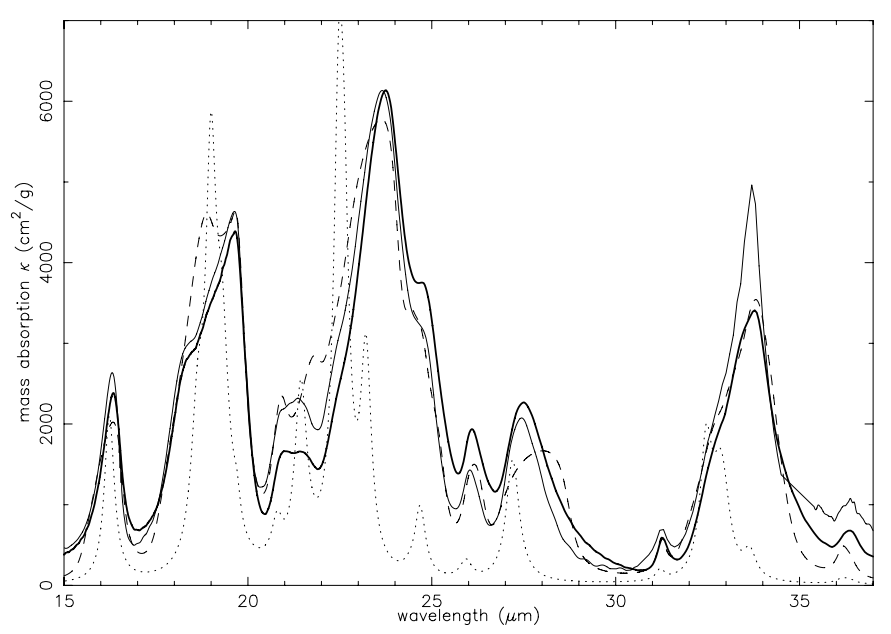

Fig. 3. The MAC spectra for different preparations of forsterite powder samples and for various shape distributions. Thick solid line: laboratory data in Koike et al. (2003). Thin solid line: laboratory data in Chihara et al. (2001). Dotted line: MAC for spheres (multiplied by 0.5 ). Dashed line: MAC for the CDE.

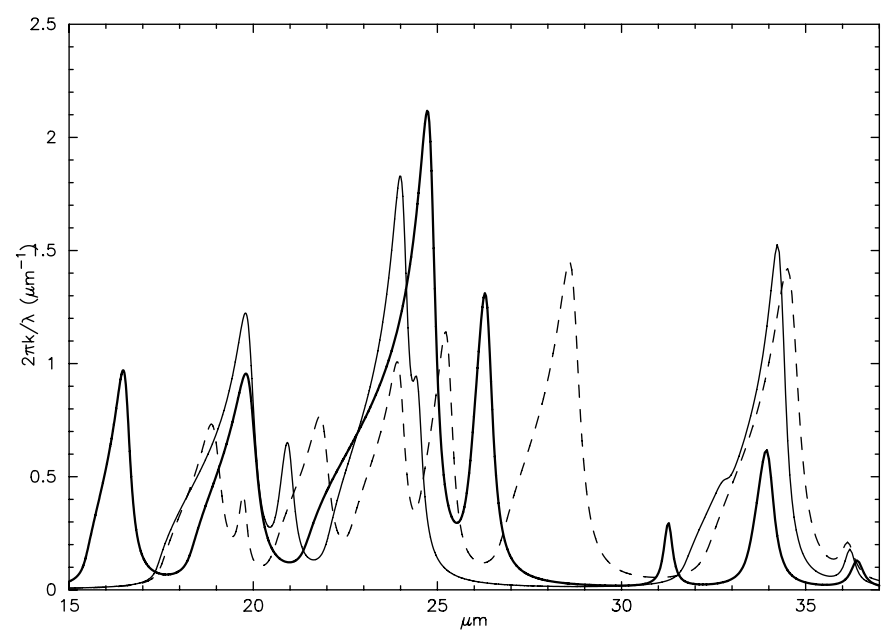

Fig. 4. Calculations of $2 \pi k / \lambda$ for three crystalline axes, where $k$ is the imaginary part of the complex index of refraction. Thick solid line: $2 \pi k / \lambda$ for the $a$-axis. Dashed line: $2 \pi k / \lambda$ for the $b$-axis. Thin solid line: $2 \pi k / \lambda$ for the $c$-axis.

refraction and is calculated from the dielectric functions. In the case of $d \sim 1 \mu \mathrm{m}$, the values of $2 \pi k d / \lambda$ become greater than 1 at the strong bands. Therefore, some particles are not in the Rayleigh limit and might influence the spectral profiles of the strong bands.

The SEM images also revealed that many particles are angular, for which an ellipsoidal approximation can give poor predictions. Furthermore, the shape distributions of the particles of the powder samples are probably not uniform. The forsterite crystal has a good cleavage plane at [010] (Takei et al. 1984). Therefore, it is plausible that grinding the forsterite crystals will easily produce the particles that are flattened perpendicular to $b$-axes of crystals rather than the particles elongated along the $b$-axes. In an astronomical situation, crystal grains might form by gas phase growth or by annealing of 
amorphous grains and might not experience destruction processes in the circumstellar environment. Therefore, one should be careful to use the laboratory ground samples as a template for cosmic dust.

Finally, we consider the far-infrared features at $\sim 50 \mu \mathrm{m}$ and $\sim 70 \mu \mathrm{m}$. The peak wavelength and the width of $70 \mu \mathrm{m}$ feature are expected to become good indicators for grain temperature (Chihara et al. 2001; Bowey et al. 2002). Absorption features of an isolated mode appear between longitudinal and transverse optical mode frequencies depending on grain shapes (Bohren \& Huffman 1983). The wavelengths of the LO and TO modes for the $50 \mu \mathrm{m}$ feature are $49.7 \mu \mathrm{m}$ and $49.8 \mu \mathrm{m}$, respectively $^{2}$. For the $70 \mu \mathrm{m}$ feature, the LO mode wavelength is $69.4 \mu \mathrm{m}$ and the TO mode wavelength is $69.7 \mu \mathrm{m}$. Because the LO-TO splittings are small for these two modes, the peak wavelengths of the features are not strongly affected by the particles shapes. However, the strength of the features can vary with the direction and the degree of the grain elongation because the vibration mode corresponding to the $50 \mu \mathrm{m}$ and $70 \mu \mathrm{m}$ features exists on $\mathrm{B}_{3 \mathrm{u}}(E \| a$-axis $)$ and $\mathrm{B}_{2 \mathrm{u}}(E \| b$-axis $)$, respectively, and no mode exists on $\mathrm{B}_{1 \mathrm{u}}(E \| c$-axis $)$ in these wavelength regions. Therefore, the ratio of the length of $a$-axis and the length of $b$-axis of the grains affects the strength of the $50 \mu \mathrm{m}$ and $70 \mu \mathrm{m}$ features and the ratio of these two features might give some constraints to the grain shapes if the optical constants that are appropriate for observed temperatures of grains are applied.

Acknowledgements. The authors thank one referee, Prof. A. M. Hofmeister, for the valuable comments that have improved the manuscript. S.T. thanks Prof. H. Nagahara for the opportunity to synthesize single crystals of forsterite and Dr. M. Morioka for his help with $\mathrm{CZ}$ synthesis of forsterite. Some of the forsterite crystal was kindly prepared by Prof. H. Takei of Osaka University. This study was supported by Grants-in-Aid from the Japan Ministry of Education, Culture, Sports, Science and Technology (12440054, 15340064).

\section{References}

Bohren, C. F., \& Huffman, D. R. 1983, Absorption and scattering of light by small particles (New York: John Wiley and Sons, Inc.)

Bowey, J. E., Barlow, M. J., Molster, F. J., et al. 2002, MNRAS, 331, L1

Cesarsky, D., Jones, A. P., Lequeux, J., \& Verstraete, L. 2000, A\&A, 358, 708

Chihara, H., Koike, C., \& Tsuchiyama, A. 2001, PASJ, 53, 243

Deer, W. A., Howie, R. A., \& Zussman, J. 1996, An Introduction to the Rock-Forming Minerals, 2nd edn. (Longman)

Fabian, D., Henning, T., Jäger, C., et al. 2001, A\&A, 378, 228

Heaton, H. I. 1971, J. Opt. Soc. Amer., 61, 275

Hofmeister, A. M. 1987, Phys. Chem. Minerals, 14, 499

Hofmeister, A. M., Keppel, E., Bowey, J. E., \& Speck, A. K. 2000, in ISO Beyond the Peaks: The 2nd ISO Workshop on Analytical Spectroscopy ed. A. Salama, M. F. Kessler, K. Leech, \& B. Schulz, ESA SP-456

Honda, M., Kataza, H., Okamoto, Y. K., et al. 2003, ApJ, 585, L59

Iishi, K. 1978, Amer. Mineral., 63, 1198

Jäger, C., Molster, F. J., Dorschner, J., et al. 1998, A\&A, 339, 904

Koike, C., \& Shibai 1994, MNRAS, 269, 1011

Koike, C., Chihara, H., Tsuchiyama, A., et al. 2003, A\&A, 399, 1101

Min, M., Hovenier, J. W., \& de Koter, A. 2003, A\&A, 404, 35

Molster, F. J., Waters, L. B. F. M., Tielens, A. G. G. M., \& Barlow, M. J. 2002, A\&A, 382, 184

Oehler, O., \& Günthard, H. Hs. 1969, J. Chem. Phys., 51, 4719

Reynard, B. 1991, Phys. Chem. Minerals, 18, 19

Servoin, J. L., \& Piriou, B. 1973, Phys. Stat. Sol. (b), 55, 677

Suto, H., Koike, C., Sogawa, H., et al. 2002, A\&A, 389, 568

Takei, H., \& Kobayashi, T. 1974, J. Crystal Growth, 23, 121

Takei, H., Hosoya, S., \& Ozima, M. 1984, in Materials Science of the Earth's Interior, ed. I. Sunagawa (Tokyo: Terra Scientific Publishing Company), 107

Waelkens, C., Waters, L. B. F. M., de Graauw, M. S., et al. 1996, A\&A, 315, L245

Waters, L. B. F. M., Molster, F. J., de Jong, T., et al. 1996, A\&A, 315, L361

${ }^{2}$ We determined the wavelengths of the LO and TO modes from the calculation of $\epsilon$. The LO mode wavelength and the TO mode wavelength are respectively determined from the peak wavelength of the imaginary part of $-1 / \epsilon$ and the peak wavelength of the imaginary part of $\epsilon$. 